\title{
GIFIHIA operator and its application to the selection of cold chain logistics enterprises
}

\author{
Shanshan Meng ${ }^{1}$ Nan Liu ${ }^{1} \cdot$ Yingdong He$^{1}$
}

Received: 27 July 2016 / Accepted: 22 January 2017 / Published online: 19 April 2017

(C) Springer International Publishing Switzerland 2017

\begin{abstract}
In this paper, we present some induced hybrid interaction averaging operators under intuitionistic fuzzy environments, including the induced hybrid interaction averaging operator and the generalized induced hybrid interaction averaging operator under intuitionistic fuzzy environments. The properties of these operators are investigated. The main advantages of these operators are that, (1) the interactions of different intuitionistic fuzzy values are taken into consideration, (2) the involved intuitionistic fuzzy values are reordered according to the induced values and then are aggregated into a collective one, (3) the attitudes of decision makers are considered by taking different values of parameter according to decision makers' preferences. We make comparisons between the results of this paper and the exsiting ones and apply the proposed operators to the selection of cold chain logistics enterprises under intuitionistic fuzzy environment. We also construct the intuitionistic fuzzy values with granularity and show the feasibility of the new approach with numerical examples.
\end{abstract}

Keywords Intuitionistic fuzzy set · Induced hybrid interaction averaging operator $\cdot$ Cold chain logistics enterprises

\section{Introduction}

As an important part of modern decision science, multiple attribute decision making has been widely used. Many

Nan Liu

nanliutju@126.com

1 College of Management and Economics, Tianjin University, Tianjin 300072, China operators focus on correctly aggregating information decision making problems have been developed on this issue (Zhao et al. 2010; Ye 2010; He et al. 2013; 2016; Beliakov et al. 2010; Merigó et al. 2011).

As a common form of decision information, Zadeh (1965) developed the fuzzy sets. To describe vague information more flexibly and practicably, Atanassov (1986) developed intuitionistic fuzzy sets (IFSs). Atanassov (1994) presented some basic operations on IFSs. Xu (2007) presented some aggregation operators under intutionistic fuzzy environments and obtained their detailed formulas with mathematical induction. Much more attention has been paid to Granular Computing and decision making problems (Xu and Yager 2006; Rodríguez et al. 2012, 2013; Chen 2014; Rodríguez et al. 2014; Pedrycz and Chen 2015; Livi and Sadeghian 2016; Chen and Chang 2015; He et al. 2015; Lorkowski and Kreinovich 2015; Chen et al. 2016a, b; Apolloni et al. 2016; Antonelli et al. 2016; Ciucci 2016; Kovalerchuk and Kreinovich 2017; Loia et al. 2016; Lingras et al. 2016; Liu and Cocea 2017; Liu et al. 2016; Maciel et al. 2016; Min and Xu 2016; Peters and Weber 2016; Skowron et al. 2016; Wilke and Portmann 2016; Xu and Wang 2016; Yao 2016; Sanchez et al. 2017; Song and Wang 2016; Wang et al. 2017; Zhou 2017). Xu and Xia (2011) dealt with financial decision making with induced generalized aggregation operators. Mendel (2016) synthesized the interval type-2 fuzzy set model for a word by comparing Hao-Mendel Approach, Interval Approach and Enhanced Interval Approach. Xu and Gou (2017) made an overview of interval-valued intuitionistic fuzzy information aggregations and applications. Wei and Zhao (2012) dealt with decision making by the induced correlated aggregating operators. Considering that distorted conclusions would be obtained if the decision makers don't take account the relationships 
among the evaluated values (Yager 2008), Wei (2012) developed some prioritized aggregation operators. Xu and Xia (2011) proposed some induced generalized intuitionistic fuzzy operators. Yager and Filev (1999) proposed the induced ordered weighted averaging operators. Chen et al. (2016a, b) proposed a multi-criteria decision making method based on the TOPSIS method under intuitionistic fuzzy environments. Apolloni et al. (2016) proposed a neurofuzzy algorithm for learning from complex granules. Dubois and Prade (2016) investigated the notion of extensional fuzzy set and highlighted its common features with the notion of formal concept in regard to a similarity relation. Das et al. (2017) developed a robust decision making method using intuitionistic fuzzy values. Chen and Tsai (2016) developed multiple attribute decision making method based on novel interval-valued intuitionistic fuzzy geometric averaging operators. Kreinovich (2016) solved systems of equations under uncertainty and explained how different practical problems lead to different mathematical and computational formulations.

Recently, some new operational laws on intuitionistic fuzzy values had been proposed in He et al. (2014a, b), which can be used in some special cases. As a good complement to the existing works, the new operational laws consider the interactions between membership function and non-membership function of different intuitionistic fuzzy values. However, when concerned with the decision making situations that the given intuitionistic fuzzy values and their ordered positions by the induced values should be considered with the interaction theory, very little work had been done. As a result, based on the works in He et al. (2014a, b), Xu (2007) and $\mathrm{Xu}$ and Yager (2006), we present some induced hybrid interaction aggregation operators on intuitionistic fuzzy values, such as the IFIHIA operator and the GIFIHIA operator. We investigate the properties of these new aggregation operators and apply them to the selection of cold chain logistics enterprises under intuitionistic fuzzy environment. The main advantage of these operators is concluded as follows, (1) the interactions of different intuitionistic fuzzy values are taken into consideration, (2) the involved intuitionistic fuzzy values are reordered according to the induced values and then are aggregated into a collective one, (3) the attitudes of decision makers are considered by taking different values of parameter according to decision makers' preferences.

The rest of the paper is organized as follows. Section 2 reviews some basic concepts. Section 3 presents someinduced hybrid interaction averaging operators under intuitionistic fuzzy environments and the corresponding properties are investigated. Section 4 investigates the selection of cold chain logistics enterprises based on the proposed operators under intuitionistic fuzzy environment. In Sect. 5, numerical examples show the feasibility and validity of the presented approach. Finally, Sect. 6 concludes the paper.

\section{Preliminaries}

Definition 1 (Atanassov 1986). Suppose that $X$ is a fixed non-empty set.

$A=\left\{\left\langle x, u_{A}(x), v_{A}(x), \pi_{A}(x)\right\rangle \mid x \in X\right\}$ indicates intuitionistic fuzzy sets (IFSs) in $X$, where $u_{A}(x), v_{A}(x) \in[0,1$, representing the membership degree and the non-membership degree respectively. $\pi_{A}(x)=1-u_{A}(x)-v_{A}(x)$, reflecting the hesitant degree of $x \in X$.

$\mathrm{Xu}(2007)$ and Xu and Yager (2006) called $A=\langle u, v\rangle$ intuitionistic fuzzy number (IFN) for computational convenience and all IFNs are denoted as IFNs(X) in this paper.

Atanassov (1994) and De et al. (2000) introduced some basic operations on IFNs, which have been widely used in multiple attribute decision making.

Let $A=\left\langle u_{A}, v_{A}\right\rangle$ be an IFN, Chen and Tan (1994) described the suitable degree of an alternative meets the decision maker's demand with score function $S(A)=u_{A}$ $v_{A}$. Hong and Choi (2000) described the accurate degree of IFN $A$ with accuracy function $H(A)=u_{A}+v_{A}$.

Based on the score function and accuracy function, $\mathrm{Xu}$ (2007) and Xu and Yager (2006) defined the comparison law for IFNs as follows.

Definition 2 Let $A=\left\langle u_{A}, v_{A}\right\rangle \in \operatorname{IFNs}(X)$ and $B=\left\langle u_{B}, v_{B}\right\rangle$ $\in \operatorname{IFNs}(X)$. Then $A<B$ if and only if.

i. $\quad S(A)<S(B)$ or

ii. $\quad S(A)=S(B)$ and $H(A)<H(B)$.

Recently, the new addition operation, scalar multiplication operation, multiplication operation and power operation are developed in $\mathrm{He}$ et al. (2014a, b) as follows.

Definition 3 Let $A=\left\langle u_{A}, v_{A}\right\rangle \in \operatorname{IFNs}(X)$ and $B=\left\langle u_{B}, v_{B}\right\rangle$ $\in \operatorname{IFNs}(X)$.

$$
\begin{aligned}
& A \hat{\oplus} B=\left\langle 1-\left(1-u_{A}\right) \cdot\left(1-u_{B}\right),\left(1-u_{A}\right) \cdot\left(1-u_{B}\right)\right. \\
&\left.-\left(1-\left(u_{A}+v_{A}\right)\right) \cdot\left(1-\left(u_{B}+v_{B}\right)\right)\right\rangle \\
& \lambda A=\left\langle 1-\left(1-u_{A}\right)^{\lambda},\left(1-u_{A}\right)^{\lambda}-\left(1-\left(u_{A}+v_{A}\right)\right)^{\lambda}\right\rangle, \lambda>0 \\
& A \hat{\otimes} B=\left\langle\left(1-v_{A}\right)\left(1-v_{B}\right)-\left(1-\left(u_{A}+v_{A}\right)\right)\right. \\
&\left.\left(1-\left(u_{B}+v_{B}\right)\right), 1-\left(1-v_{A}\right)\left(1-v_{B}\right)\right\rangle \\
& A^{\lambda}=\left\langle\left(1-v_{A}\right)^{\lambda}-\left(1-\left(u_{A}+v_{A}\right)\right)^{\lambda}, 1-\left(1-v_{A}\right)^{\lambda}\right\rangle, \lambda>0
\end{aligned}
$$




\section{Intuitionistic fuzzy induced hybrid interaction averaging (IFIHIA) operator}

Definition 4 Let $\quad A_{i}=\left\langle u_{A_{i}}, v_{A_{i}}\right\rangle \in \operatorname{IFNs}(X)$ $(i=1,2, \ldots, n) . p_{i}(i=1,2, \ldots, n)$ is the induced value. The IFIHIA operator is defined as

$\operatorname{IFIHIA}_{\omega, w}\left(A_{1}, \ldots, A_{n}\right)=\hat{\oplus}_{i=1}^{n} w_{i} \tilde{A}_{\text {index }(i)}$,

where $\omega=\left(\omega_{1}, \ldots \omega_{n}\right)^{T}$ is the weight vector of $A_{i} \quad(i=1,2, \ldots, n)$, satisfying $\omega_{i} \in\left[0,1\right.$ and $\sum_{i=1}^{n} \omega_{i}=1$, $\tilde{A}_{i}$ denotes $n \omega_{i} A_{i}(i=1, \ldots, n), \tilde{A}_{i}$ is reordered according to $p_{i}$ as $\tilde{A}_{\text {index(i) }}(i=1,2, \ldots, n), w=\left(w_{1}, w_{2}, \ldots, w_{n}\right)^{T}$ is the weight vector of $\tilde{A}_{\text {index }(i)}(i=1,2, \ldots, n)$, satisfying $w_{i} \in\left[0,1\right.$ and $\sum_{i=1}^{n} w_{i}=1$.

Theorem $1 \quad$ Let $A_{i}=\left\langle u_{A_{i}}, v_{A_{i}}\right\rangle \in \operatorname{IFNs}(X) \quad(i=1,2, \ldots, n)$. $p_{i}(i=1,2, \ldots, n)$ is the induced value. Then

$$
\begin{aligned}
& \operatorname{IFIHIA}_{\omega, w}\left(A_{1}, \ldots, A_{n}\right)=\left\langle 1-\prod_{i=1}^{n}\left(1-u_{\tilde{A}_{\text {index }(i)}}\right)^{w_{i}},\right. \\
& \left.\prod_{i=1}^{n}\left(1-u_{\tilde{A}_{\text {index }(i)}}\right)^{w_{i}}-\prod_{i=1}^{n}\left(1-\left(u_{\tilde{A}_{\text {index }(i)}}+v_{\tilde{A}_{\text {index }(i)}}\right)\right)^{w_{i}}\right\rangle
\end{aligned}
$$

And, IFIHIA ${ }_{\omega, w}\left(A_{1}, \ldots, A_{n}\right) \in \operatorname{IFNs}(X)$.

Theorem 2 (Idempotency) Let $A_{i}=\left\langle u_{A_{i}}, v_{A_{i}}\right\rangle$ $(i=1,2, \ldots, n)$ be a collection of IFNs, if all $A_{i}$ are equal, supposed as $A$, then IFIHIA $A_{\omega, w}\left(A_{1}, \ldots, A_{n}\right)=A$.

Theorem 3 (Commutativity). Let $A_{i}=\left\langle u_{A_{i}}, v_{A_{i}}\right\rangle$ $(i=1,2, \ldots, n)$ be a collection of IFNs, if $A_{i}^{\prime}(i=1, \ldots n)$ is any permutation of $A_{i}(i=1, \ldots n)$, then

$$
\operatorname{IFIHI}_{\omega, w}\left(A_{1}, \ldots, A_{n}\right)=\operatorname{IFIHI} A_{\omega, w}\left(A_{1}^{\prime}, \ldots, A_{n}^{\prime}\right) .
$$

Example $\quad 1$ Let $\quad A_{1}=\langle 0.2,0.7\rangle A_{2}=\langle 0.5,0.3\rangle$ $A_{3}=\langle 0.4,0.5\rangle A_{4}=\langle 0.3,0.4\rangle A_{5}=\langle 0.6,0\rangle$ be five IFNs. $\omega=\{0.25,0.20,0.15,0.18,0.22\}^{T}$ is the weight vector of $A_{i} \quad(i=1,2, \ldots, 5)$.

By the operational law in Atanassov (1986), De et al. (2000) and $\mathrm{Xu}(2007)$, it has.

$$
\tilde{A}_{1}=\left\langle 1-(1-0.2)^{5 \times 0.25}, 0.7^{5 \times 0.25}\right\rangle=\langle 0.243,0.640\rangle,
$$

$\tilde{A}_{2}=\left\langle 1-(1-0.5)^{5 \times 0.20}, 0.3^{5 \times 0.20}\right\rangle=\langle 0.500,0.300\rangle$,

$\tilde{A}_{3}=\left\langle 1-(1-0.4)^{5 \times 0.15}, 0.5^{5 \times 0.15}\right\rangle=\langle 0.318,0.595\rangle$,

$\tilde{A}_{4}=\left\langle 1-(1-0.3)^{5 \times 0.18}, 0.4^{5 \times 0.18}\right\rangle=\langle 0.275,0.438\rangle$,

$\tilde{A}_{5}=\left\langle 1-(1-0.6)^{5 \times 0.22}, 0^{5 \times 0.22}\right\rangle=\langle 0.635,0.00\rangle$.

By Definition 2, we have

$S\left(\tilde{A}_{1}\right)=-0.3969, S\left(\tilde{A}_{2}\right)=0.2000, S\left(\tilde{A}_{3}\right)=-0.2763$,

$S\left(\tilde{A}_{4}\right)=-0.1639, S\left(\tilde{A}_{5}\right)=0.6350$.

For a fair comparison, we suppose the score values of IFNs are the induced values, Obviously,

$S\left(\tilde{A}_{5}\right)>S\left(\tilde{A}_{2}\right)>S\left(\tilde{A}_{4}\right)>S\left(\tilde{A}_{3}\right)>S\left(\tilde{A}_{1}\right)$.

So

$\tilde{A}_{\text {index }(1)}=\langle 0.6350,0.0000\rangle, \tilde{A}_{\text {index }(2)}=\langle 0.5000,0.3000\rangle$,

$\tilde{A}_{\text {index(3) }}=\langle 0.2745,0.4384\rangle, \tilde{A}_{\text {index }(4)}=\langle 0.3183,0.5946\rangle$,

$\tilde{A}_{\text {index }(5)}=\langle 0.2434,0.6403\rangle$.

Suppose that $w=(0.112,0.236,0.304,0.236,0.112)^{T}$. Then, by the intuitionistic fuzzy hybrid interaction averaging (IFHA) operator in $\mathrm{Xu}$ (2007), it follows that.

$$
A=I F H A_{w, \omega}\left(A_{1}, A_{2}, A_{3}, A_{4}, A_{5}\right)=\left\langle 1-\prod_{j=1}^{5}\left(1-u_{\tilde{A}_{\sigma()}}\right)^{w_{j}},\right.
$$

$\left.\prod_{j=1}^{5}\left(v_{\tilde{A}_{\sigma(j)}}\right)^{w_{i}}\right\rangle=\left\langle 1-(1-0.6350)^{0.112} \cdot(1-0.5)^{0.236}\right.$.

$\cdot(1-0.2745)^{0.304} \cdot(1-0.3183)^{0.236} \cdot(1-0.2434)^{0.112}$, $\left.0.1703^{0.112} \cdot 0.3^{0.236} \cdot 0.4384^{0.304} \cdot 0.5946^{0.236} \cdot 0.0000^{0.112}\right\rangle$ $=\langle 0.3910,0.000\rangle$.

While according to Definition 3, we have

$$
\begin{aligned}
& \tilde{A}_{1}=\left\langle 1-(1-0.2)^{5 \times 0.25},(1-0.2)^{5 \times 0.25}-(1-0.9)^{5 \times 0.25}\right\rangle=\langle 0.2434,0.7004\rangle, \\
& \tilde{A}_{2}=\left\langle 1-(1-0.5)^{5 \times 0.20},(1-0.5)^{5 \times 0.20}-(1-0.8)^{5 \times 0.20}\right\rangle=\langle 0.500,0.300\rangle, \\
& \tilde{A}_{3}=\left\langle 1-(1-0.4)^{5 \times 0.15},(1-0.4)^{5 \times 0.15}-(1-0.9)^{5 \times 0.15}\right\rangle=\langle 0.3183,0.5039\rangle, \\
& \tilde{A}_{4}=\left\langle 1-(1-0.3)^{5 \times 0.18},(1-0.3)^{5 \times 0.18}-(1-0.7)^{5 \times 0.18}\right\rangle=\langle 0.2745,0.3870\rangle, \\
& \tilde{A}_{5}=\left\langle 1-(1-0.6)^{5 \times 0.22},(1-0.6)^{5 \times 0.22}-(1-0.6)^{5 \times 0.22}\right\rangle=\langle 0.6350,0.0000\rangle .
\end{aligned}
$$


According to Definition 2, we obtain

$S\left(\tilde{A}_{1}\right)=-0.4570, S\left(\tilde{A}_{2}\right)=0.2000, S\left(\tilde{A}_{3}\right)=-0.1856$,

$S\left(\tilde{A}_{4}\right)=-0.1125, S\left(\tilde{A}_{5}\right)=0.6350$.

obviously,

$S\left(\tilde{A}_{5}\right)>S\left(\tilde{A}_{2}\right)>S\left(\tilde{A}_{4}\right)>S\left(\tilde{A}_{3}\right)>S\left(\tilde{A}_{1}\right)$.

Thus,

$\tilde{A}_{\text {index }(1)}=\langle 0.6350,0.0000\rangle, \tilde{A}_{\text {index }(2)}=\langle 0.5000,0.3000\rangle$,

$\tilde{A}_{\text {index }(3)}=\langle 0.2745,0.3870\rangle, \tilde{A}_{\text {index }(4)}=\langle 0.3183,0.5039\rangle$,

$\tilde{A}_{\text {index }(5)}=\langle 0.2434,0.7004\rangle$.

Suppose that $w=(0.112,0.236,0.304,0.236,0.112)^{T}$, which is determined by the normal distribution based method (Xu 2007). Then, by Theorem 1, it follows that is a mapping GIFIHIA ${ }_{\lambda}:$ IFN $^{n} \rightarrow$ IFN, which has an associated vector $w=\left(w_{1}, w_{2}, \ldots, w_{n}\right)^{T}$, satisfying $w_{i} \in[0,1$ and $\sum_{i=1}^{n} w_{i}=1$ such that

$\operatorname{GIFIHIA}_{\lambda}\left(A_{1}, \ldots, A_{n}\right)=\left(\hat{\oplus}_{i=1}^{n} w_{i} \tilde{A}_{i n d e x(i)}^{\lambda}\right)^{1 / \lambda}$,

where $\omega=\left(\omega_{1}, \ldots \omega_{n}\right)^{T}$ is the weight vector of $A_{i} \quad(i=1,2, \ldots, n)$, satisfying $\omega_{i} \in\left[0,1\right.$ and $\sum_{i=1}^{n} \omega_{i}=1$, $\tilde{A}_{i}$ denotes $n \omega_{i} A_{i}(i=1,2, \ldots, n), n$ is the balancing coefficient, which plays a role of balance. $\tilde{A}_{i}$ is reordered according to $p_{i}$ as $\tilde{A}_{\text {index }(i)}(i=1,2, \ldots, n)$.

$$
\begin{aligned}
I A= & I_{F I H I A} A_{w, \omega}\left(A_{1}, A_{2}, A_{3}, A_{4}, A_{5}\right)=\left\langle 1-\prod_{j=1}^{5}\left(1-u_{\tilde{A}_{\text {index(j) }}}\right)^{w_{j}}, \prod_{j=1}^{5}\left[1-u_{\tilde{A}_{\text {index(j) }}}\right]^{w_{i}}-\prod_{j=1}^{5}\left[1-\left(u_{\tilde{A}_{\text {index(j) }}}+v_{\tilde{\tilde{A}}_{\text {index(j) }}}\right)\right]^{w_{i}}\right\rangle \\
= & \left\langle 1-(1-0.6350)^{0.112} \cdot(1-0.5)^{0.236} \cdot(1-0.2750)^{0.304} \cdot(1-0.3183)^{0.236} \cdot(1-0.2434)^{0.112}, 0.6090-0.1703^{0.112}\right. \\
& \left.\cdot 0.2^{0.236} \cdot 0.338^{0.304} \cdot 0.1778^{0.236} \cdot 0.0562^{0.112}\right\rangle=\langle 0.3910,0.3950\rangle .
\end{aligned}
$$

It is obvious that $v_{\text {IFIHA }}\left(A_{1}, \ldots, A_{n}\right)=0.3950 \neq 0$. Thus, $v_{A_{5}}$ doesn't play a decisive role, which is the advantage of the proposed operator in this paper over that in $\mathrm{Xu}$ (2007).

The IFIHIA operator can be interpreted from three aspects as follows.

1. It considers not only the effects of membership function of different IFNs and effects of non-membership degree of different IFN $s$, but also the interactions of different IFNs.

2. The weighted IFNs $n \omega_{i} A_{i}(i=1, \ldots, n)$ are reordered according to the induced value $p_{i}(i=1,2, \ldots, n)$. The weighted IFNs $n \omega_{i} A_{i}(i=1, \ldots, n)$ are obtained by multiplies $A_{i}(i=1, \ldots, n)$ by the corresponding weights $\omega=\left(\omega_{1}, \ldots \omega_{n}\right)^{T}$ and a balancing coefficient $n$.

3. In the process of all the weighted IFNs $w_{i} \tilde{A}_{\text {index }(i)}(i=1,2, \ldots, n)$ are aggregated into a collective one, both the given IFNs and their induced values $p_{i}(i=1,2, \ldots, n)$ are considered.

\section{Generalized intuitionistic fuzzy induced hybrid interaction averaging (GIFIHIA) operator}

Definition 5 Let $A_{i}=\left\langle u_{A_{i}}, v_{A_{i}}\right\rangle \quad(i=1,2, \ldots, n)$ be a collection of IFNs, $\lambda>0, p_{i}(i=1,2, \ldots, n)$ be the induced value. The generalized intuitionistic fuzzy induced hybrid interaction averaging (GIFIHIA) operator of dimension $n$
Lemma 1 Let $A_{i}=\left\langle u_{A_{i}}, v_{A_{i}}\right\rangle(i=1,2, \ldots, n)$ be a collection of IFNs. Then

$$
\begin{aligned}
\hat{\oplus}_{i=1}^{n} w_{i} A_{i}^{\lambda}= & \left\langle 1-\prod_{i=1}^{n}\left(1-\left(1-v_{A_{i}}\right)^{\lambda}+\left(1-\left(u_{A_{i}}+v_{A_{i}}\right)\right)^{\lambda}\right)^{w_{i}},\right. \\
& \prod_{i=1}^{n}\left(1-\left(1-v_{A_{i}}\right)^{\lambda}+\left(1-\left(u_{A_{i}}+v_{A_{i}}\right)\right)^{\lambda}\right)^{w_{i}} \\
& \left.-\prod_{i=1}^{n}\left(1-\left(u_{A_{i}}+v_{A_{i}}\right)\right)^{\lambda w_{i}}\right\rangle
\end{aligned}
$$

where $w=\left(w_{1}, w_{2}, \ldots, w_{n}\right)^{T}$ is the weighting vector of $A_{i}$, satisfying $w_{i} \in[0,1$ and $>$.

Proof By using mathematical induction on $n$, we prove Lemma 1 as follows.

when $n=1, w_{1}=1$, we obtain

$w_{1} A_{1}{ }^{\lambda}=A_{1}{ }^{\lambda}=\left\langle\left(1-v_{A_{1}}\right)^{\lambda}-\left(1-\left(u_{A_{1}}+v_{A_{1}}\right)\right)^{\lambda}\right.$,

$\left.1-\left(1-v_{A_{1}}\right)^{\lambda}\right\rangle=\left\langle 1-\left(1-\left(1-v_{A_{1}}\right)^{\lambda}+\left(1-\left(u_{A_{1}}+v_{A_{1}}\right)\right)^{\lambda}\right)^{1}\right.$,

$\left.\left(1-\left(1-v_{A_{1}}\right)^{\lambda}+\left(1-\left(u_{A_{1}}+v_{A_{1}}\right)\right)^{\lambda}\right)^{1}-\left(1-\left(u_{A_{1}}+v_{A_{1}}\right)\right)^{1 \cdot \lambda}\right\rangle$

$=\left\langle 1-\left(1-\left(1-v_{A_{1}}\right)^{\lambda}+\left(1-\left(u_{A_{1}}+v_{A_{1}}\right)\right)^{\lambda}\right)^{w_{1}}\right.$,

$\left.\left(1-\left(1-v_{A_{1}}\right)^{\lambda}+\left(1-\left(u_{A_{1}}+v_{A_{1}}\right)\right)^{\lambda}\right)^{w_{1}}-\left(1-\left(u_{A_{1}}+v_{A_{1}}\right)\right)^{\lambda \cdot w_{1}}\right\rangle$.

Thus, Lemma 1 is established for $n=1$.

If Lemma 1 holds for $n=k$. Then, $n=k+1$, by inductive assumption and Eq. (1), we get 


$$
\begin{aligned}
& l \hat{\oplus}_{i=1}^{k+1} w_{i} A_{i}^{\lambda}=\left(\hat{\oplus}_{i=1}^{k} w_{i} A_{i}^{\lambda}\right) \hat{\oplus} w_{k+1} A_{k+1}^{\lambda} \\
& \quad=\left\langle 1-\prod_{i=1}^{k}\left(1-\left(1-v_{A_{i}}\right)^{\lambda}+\left(1-\left(u_{A_{i}}+v_{A_{i}}\right)\right)^{\lambda}\right)^{w_{i}}, \prod_{i=1}^{k}\left(1-\left(1-v_{A_{i}}\right)^{\lambda}+\left(1-\left(u_{A_{i}}+v_{A_{i}}\right)\right)^{\lambda}\right)^{w_{i}}-\prod_{i=1}^{k}\left(1-\left(u_{A_{i}}+v_{A_{i}}\right)\right)^{\lambda w_{i}}\right\rangle \\
& \quad \hat{\oplus}\left\langle 1-\left(1-\left(1-v_{A_{k+1}}\right)^{\lambda}+\left(1-\left(u_{A_{k+1}}+v_{A_{k+1}}\right)\right)^{\lambda}\right)^{w_{k+1}},\left(1-\left(1-v_{A_{k+1}}\right)^{\lambda}+\left(1-\left(u_{A_{k+1}}+v_{A_{k+1}}\right)\right)^{\lambda}\right)^{w_{k+1}}-\left(1-\left(u_{A_{k+1}}+v_{A_{k+1}}\right)\right)^{\lambda \cdot w_{k+1}}\right\rangle \\
& =\left\langle 1-\prod_{i=1}^{k+1}\left(1-\left(1-v_{A_{i}}\right)^{\lambda}+\left(1-\left(u_{A_{i}}+v_{A_{i}}\right)\right)^{\lambda}\right)^{w_{i}}, \prod_{i=1}^{k+1}\left(1-\left(1-v_{A_{i}}\right)^{\lambda}+\left(1-\left(u_{A_{i}}+v_{A_{i}}\right)\right)^{\lambda}\right)^{w_{i}}-\prod_{i=1}^{k+1}\left(1-\left(u_{A_{i}}+v_{A_{i}}\right)\right)^{\lambda w_{i}}\right\rangle
\end{aligned}
$$

i.e. Lemma 1 is established for $n=k+1$.

Thus, Lemma 1 holds for all $n$ with the mathematical induction on $n$.

Theorem 4 Let $A_{i}=\left\langle u_{A_{i}}, v_{A_{i}}\right\rangle \quad(i=1,2, \ldots, n)$ be a collection of IFNs. $\lambda>0$. Then $\operatorname{GIFIHIA}_{\lambda}\left(A_{1}, A_{2}, \ldots A_{n}\right) \in$ $\operatorname{IFNs}(X)$. Furthermore,
Therefore, Eq. (8) is established.

Next, we prove the result that $\operatorname{GIFIHIA}_{\lambda}\left(A_{1}, A_{2}, \ldots A_{n}\right) \in \operatorname{IFNs}(X)$.

Let GIFIHIA $\lambda_{\lambda}\left(A_{1}, A_{2}, \ldots A_{n}\right)=E=\left\langle u_{E}, v_{E}\right\rangle$, By Eq. (8), we have

$$
\begin{aligned}
& \operatorname{GIFIHIA}_{\lambda}\left(A_{1}, A_{2}, \ldots A_{n}\right)=\left\langle\left(1-\prod_{i=1}^{n}\left(1-\left(1-v_{\tilde{A}_{\text {index }(i)}}\right)^{\lambda}+\left(1-\left(u_{\tilde{A}_{\text {index }(i)}}+v_{\tilde{A}_{\text {index }(i)}}\right)\right)^{\lambda}\right)^{w_{i}}+\prod_{i=1}^{n}\left(1-\left(u_{\tilde{A}_{\text {index }(i)}}+v_{\tilde{A}_{\text {index }(i)}}\right)\right)^{\lambda \cdot w_{i}}\right)^{1 / \lambda}\right. \\
& \left.-\prod_{i=1}^{n}\left(1-\left(u_{\tilde{A}_{\text {index }(i)}}+v_{\tilde{A}_{\text {index }(i)}}\right)\right)^{w_{i}}, 1-\left(1-\prod_{i=1}^{n}\left(1-\left(1-v_{\tilde{A}_{\text {index }(i)}}\right)^{\lambda}+\left(1-\left(u_{\tilde{A}_{\text {inder }(i)}}+v_{\tilde{A}_{\text {index }(i)}}\right)\right)^{\lambda}\right)^{w_{i}}+\prod_{i=1}^{n}\left(1-\left(u_{\tilde{A}_{\text {inder }(i)}}+v_{\tilde{A}_{\text {index }(i)}}\right)\right)^{\lambda w_{i}}\right)^{1 / \lambda}\right\rangle .
\end{aligned}
$$

Proof By Lemma 1 we get

$$
\begin{aligned}
& \hat{\oplus}_{i=1}^{n} w_{i} \tilde{A}_{\text {index }(i)}^{\lambda}=\left\langle 1-\prod_{i=1}^{n}\left(1-\left(1-v_{\tilde{A}_{\text {index }(i)}}\right)^{\lambda}+\left(1-\left(u_{\tilde{A}_{\text {index }(i)}}+v_{\tilde{A}_{\text {index }(i)}}\right)\right)^{\lambda}\right)^{w_{i}}\right. \\
& \left.\quad \prod_{i=1}^{n}\left(1-\left(1-v_{\tilde{A}_{\text {index }(i)}}\right)^{\lambda}+\left(1-\left(u_{\tilde{A}_{\text {index }(i)}}+v_{\tilde{A}_{\text {index }(i)}}\right)\right)^{\lambda}\right)^{w_{i}}-\prod_{i=1}^{n}\left(1-\left(u_{\tilde{A}_{\text {index }(i)}}+v_{\tilde{A}_{\text {index }(i)}}\right)\right)^{\lambda w_{i}}\right\rangle .
\end{aligned}
$$

Then according to Eqs. (4) and (7), we have

$$
\begin{aligned}
& \operatorname{GIFIHIA}_{\lambda}\left(A_{1}, A_{2}, \ldots A_{n}\right)=\left(\hat{\oplus}_{i=1}^{n} w_{i}\left(\tilde{A}_{\text {index }(i)}\right)^{\lambda}\right)^{1 / \lambda} \\
& =\left\langle\left(1-\prod_{i=1}^{n}\left(1-\left(1-v_{\tilde{A}_{\text {index }(i)}}\right)^{\lambda}+\left(1-\left(u_{\tilde{A}_{\text {index }(i)}}+v_{\tilde{A}_{\text {index }(i)}}\right)\right)^{\lambda}\right)^{w_{i}}+\prod_{i=1}^{n}\left(1-\left(u_{\tilde{A}_{\text {inder }(i)}}+v_{\tilde{A}_{\text {index(i) }}}\right)\right)^{\lambda \cdot w_{i}}\right)^{1 / \lambda}\right. \\
& \left.-\prod_{i=1}^{n}\left(1-\left(u_{\tilde{A}_{\text {index }(i)}}+v_{\tilde{A}_{\text {index }(i)}}\right)\right)^{w_{i}}, 1-\left(1-\prod_{i=1}^{n}\left(1-\left(1-v_{\tilde{A}_{\text {index }(i)}}\right)^{\lambda}+\left(1-\left(u_{\tilde{A}_{\text {index }(i)}}+v_{\tilde{A}_{\text {index }(i)}}\right)\right)^{\lambda}\right)^{w_{i}}+\prod_{i=1}^{n}\left(1-\left(u_{\tilde{A}_{\text {index }(i)}}+v_{\tilde{A}_{\text {index }(i)}}\right)\right)^{\lambda w_{i}}\right)^{1 / \lambda}\right\rangle^{1 / 2}
\end{aligned}
$$




$$
\begin{aligned}
& v_{E}=1-\left(1-\prod_{i=1}^{n}\left(1-\left(1-v_{\tilde{A}_{\text {index }(i)}}\right)^{\lambda}+\left(1-\left(u_{\tilde{A}_{\text {inder }(i)}}+v_{\tilde{A}_{\text {index }(i)}}\right)\right)^{\lambda}\right)^{w}\right. \\
& \left.+\prod_{i=1}^{n}\left(1-\left(u_{\tilde{A}_{\text {index }(i)}}+v_{\tilde{A}_{\text {index }(i)}}\right)\right)^{\lambda w_{i}}\right)^{1 / \lambda}, \\
& u_{E} \geqslant\left(0+\prod_{i=1}^{n}\left(1-\left(u_{\tilde{A}_{\text {index }(i)}}+v_{\tilde{A}_{\text {index }(i)}}\right)\right)^{\lambda \cdot w_{i}}\right)^{1 / \lambda} \\
& -\prod_{i=1}^{n}\left(1-\left(u_{\tilde{A}_{\text {index }(i)}}+v_{\tilde{A}_{\text {index }(i)}}\right)\right)^{w_{i}}=0 . \\
& u_{E}=\left(\begin{array}{r}
1-\prod_{i=1}^{n}\left(1-\left(1-v_{\tilde{A}_{\text {index }(i)}}\right)^{\lambda}+\left(1-\left(u_{\tilde{A}_{\text {index }(i)}}+v_{\tilde{A}_{\text {index }(i)}}\right)\right)^{\lambda}\right)^{w_{i}} \\
+\prod_{i=1}^{1 / \lambda}\left(1-\left(u_{\tilde{A}_{\text {index }(i)}}+v_{\tilde{A}_{\text {index }(i)}}\right)\right)^{\lambda \cdot w_{i}}
\end{array} \prod^{n}\left(1-\left(u_{\tilde{A}_{\text {index }(i)}}+v_{\tilde{A}_{\text {index }(i)}}\right)\right)^{w_{i}}\right.
\end{aligned}
$$

According to Definition 1, we have And $0 \leqslant 1-\left(1-v_{\tilde{A}_{\sigma(i)}}\right)^{\lambda} \leqslant 1$, then

$v_{E} \geqslant 1-\left(1-\prod_{i=1}^{n}\left(0+\left(1-\left(u_{\tilde{A}_{\text {index }(i)}}+v_{\tilde{A}_{\text {index }(i)}}\right)\right)^{\lambda}\right)^{w_{i}}\right.$

$\left.+\prod_{i=1}^{n}\left(1-\left(u_{\tilde{A}_{\text {index }(i)}}+v_{\tilde{A}_{\text {index }(i)}}\right)\right)^{\lambda w_{i}}\right)^{1 / \lambda}=0$.

And

$$
\begin{aligned}
1- & \left(1-v_{\tilde{A}_{\text {index }(i)}}\right)^{\lambda}+\left(1-\left(u_{\tilde{A}_{\text {index }(i)}}+v_{\tilde{A}_{\text {index }(i)}}\right)\right)^{\lambda} \\
& =1-\left(\left(1-v_{\tilde{A}_{\text {index }(i)}}\right)^{\lambda}-\left(1-\left(u_{\tilde{A}_{\text {index }(i)}}+v_{\tilde{A}_{\text {index }(i)}}\right)\right)^{\lambda}\right) \in[0,1] .
\end{aligned}
$$

$$
\begin{aligned}
& \left(\prod_{i=1}^{n}\left(1-\left(1-v_{\tilde{A}_{\text {index }(i)}}\right)^{\lambda}+\left(1-\left(u_{\tilde{A}_{\text {index }(i)}}+v_{\tilde{A}_{\text {index }(i)}}\right)\right)^{\lambda}\right)^{w_{i}}\right. \\
& \left.\quad-\prod_{i=1}^{n}\left(1-\left(u_{\tilde{A}_{\text {index }(i)}}+v_{\tilde{A}_{\text {index }(i)}}\right)\right)^{\lambda \cdot w_{i}}\right) \\
& \quad \geqslant \prod_{i=1}^{n}\left(0+\left(1-\left(u_{\tilde{A}_{\text {index }(i)}}+v_{\tilde{A}_{\text {index }(i)}}\right)\right)^{\lambda}\right)^{w_{i}} \\
& \quad-\prod_{i=1}^{n}\left(1-\left(u_{\tilde{A}_{\text {index }(i)}}+v_{\tilde{A}_{\text {index }(i)}}\right)\right)^{\lambda \cdot w_{i}}=0,
\end{aligned}
$$

Thus,

$$
\begin{aligned}
v_{E} & \leq 1-\left(1-\prod_{i=1}^{n}(1)^{w_{i}}+\prod_{i=1}^{n}\left(1-\left(u_{\tilde{A}_{\text {index }(i)}}+v_{\tilde{A}_{\text {index }(i)}}\right)\right)^{\lambda w_{i}}\right)^{1 / \lambda}=1-\left(1-1+\prod_{i=1}^{n}\left(1-\left(u_{\tilde{A}_{\text {index }(i)}}+v_{\tilde{A}_{\text {index }(i)}}\right)\right)^{\lambda w_{i}}\right)^{1 / \lambda} \\
& =1-\left(\prod_{i=1}^{n}\left(1-\left(u_{\tilde{A}_{\text {index }(i)}}+v_{\tilde{A}_{\text {index }(i)}}\right)\right)^{\lambda w_{i}}\right)^{1 / \lambda}=1-\left(\prod_{i=1}^{n}\left(1-\left(u_{\tilde{A}_{\text {index }(i)}}+v_{\tilde{A}_{\text {index }(i)}}\right)\right)^{w_{i}}\right) \leq 1
\end{aligned}
$$

By Eqs. (11) and (13), we have

$0 \leqslant v_{E} \leqslant 1$.

By Eq. (12) and $0 \leqslant w_{i} \leqslant 1$, we obtain $\left(1-\left(1-v_{\tilde{A}_{\text {index }(i)}}\right)^{\lambda}+\left(1-\left(u_{\tilde{A}_{\text {index }(i)}}+v_{\tilde{A}_{\text {index }(i)}}\right)\right)^{\lambda}\right)^{w_{i}} \in[0,1]$,

Thus,

$$
\begin{aligned}
1 & -\prod_{i=1}^{n}\left(1-\left(1-v_{\tilde{A}_{\text {index }(i)}}\right)^{\lambda}\right. \\
& \left.+\left(1-\left(u_{\tilde{A}_{\text {index }(i)}}+v_{\tilde{A}_{\text {index }(i)}}\right)\right)^{\lambda}\right)^{w_{i}} \in[0,1] .
\end{aligned}
$$

By Eqs. (10) and (15), we have

$$
\begin{aligned}
u_{E} & \leqslant(1-0)^{1 / \lambda}-\prod_{i=1}^{n}\left(1-\left(u_{\tilde{A}_{\text {index }(i)}}+v_{\tilde{A}_{\text {index }(i)}}\right)\right)^{w_{i}} \\
& =1-\prod_{i=1}^{n}\left(1-\left(u_{\tilde{A}_{\text {index }(i)}}+v_{\tilde{A}_{\text {index }(i)}}\right)\right)^{w_{i}} \leqslant 1 .
\end{aligned}
$$

Then by Eqs. (16) and (18), we obtain

$0 \leq u_{E} \leq 1$.

According to Eqs. (9) and (10), we have

$$
u_{E}+v_{E}=1-\prod_{i=1}^{n}\left(1-\left(u_{\tilde{A}_{\text {index }(i)}}+v_{\tilde{A}_{\text {index }(i)}}\right)\right)^{w_{i}} \in[0,1] .
$$


Therefore, according to Eqs. (14), (19) and (20) and Definition 1 , we have

$\operatorname{GIFIHIA}_{\lambda}\left(A_{1}, A_{2}, \ldots A_{n}\right) \in \operatorname{IFNs}(X)$.

Theorem 5 Let $A_{i}=\left\langle u_{A_{i}}, v_{A_{i}}\right\rangle \quad(i=1,2, \ldots, n)$ be a collection of IFNs, $\lambda>0 . \lambda \rightarrow 0$. Then the GIFIHIA operator approaches the following limit.
Theorem 7 (Commutativity) Let $A_{i}=\left\langle u_{A_{i}}, v_{A_{i}}\right\rangle$ $(i=1,2, \ldots, n)$ be a collection of IFNs. $\lambda>0$. $w=\left(w_{1}, w_{2}, \ldots, w_{n}\right)^{T}$ is the weighting vector of $A_{i} \quad$ and $\quad w_{i}=\frac{1}{n}(i=1, \ldots, n)$. If $\quad\left(A^{\prime}{ }_{1}, A^{\prime}{ }_{2}, \ldots A^{\prime}{ }_{n}\right)$ is any permutation of $\left(A_{1}, A_{2}, \ldots A_{n}\right)$, then $\operatorname{GIFIHIA}_{\lambda}\left(A_{1}, A_{2}, \ldots A_{n}\right)=\operatorname{GIFIHIA}_{\lambda}\left(A_{1}^{\prime}, A_{2}^{\prime}, \ldots A_{n}^{\prime}\right)$.

$$
\begin{aligned}
& \operatorname{Lim}_{\lambda \rightarrow 0} \operatorname{GIFIHIA}_{\lambda}\left(A_{1}, \cdots A_{n}\right)=\left\langle e^{\sum_{i=1}^{n}\left(w_{i}\left(\ln \left(1-v_{\tilde{A}_{\text {index }}(i)}\right)-\ln \left(1-\left(u_{\tilde{A}_{\text {index }(i)}}+v_{\tilde{A}_{\text {index }}(i)}\right)\right)\right)+\ln \left(\prod_{i=1}^{n}\left(1-\left(u_{\tilde{A}_{\text {index }(i)}}+v_{\tilde{A}_{\text {index }}(i)}\right)\right)^{w_{i}}\right)\right.}\right.
\end{aligned}
$$

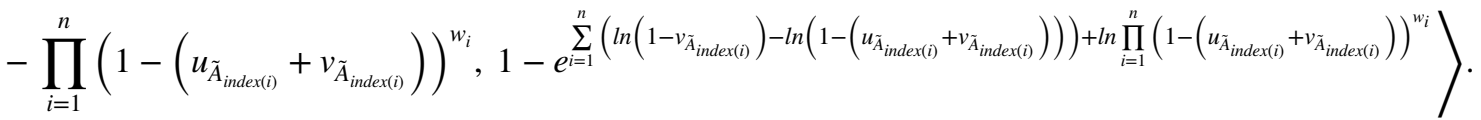

$$
\text { And } \operatorname{Lim}_{\lambda \rightarrow 0} \operatorname{GIFIHIA}_{\lambda}\left(A_{1}, A_{2}, \ldots A_{n}\right) \in \operatorname{IFNs}(X) \text {. }
$$

Proof Similar to He et al. (2014b) and omitted here.

Theorem 6 (Idempotency) $\operatorname{Let}_{i}=\left\langle u_{A_{i}}, v_{A_{i}}\right\rangle \quad(i=1,2$, $\ldots, n)$ be a collection of IFNs, $\lambda>0 . w=\left(w_{1}, w_{2}, \ldots, w_{n}\right)^{T}$ is the weighting vector of $A_{i}$, satisfying $w_{i} \in[0,1$ and $>$. If all $A_{i}$ are equal, denoted as $A$, and $\omega=\left(\frac{1}{n}, \cdots \frac{1}{n}\right)$, then $\operatorname{GIFIHIA}_{\lambda}\left(A_{1}, A_{2}, \ldots A_{n}\right)=A$.

Proof Let $A_{i}=A=\left\langle u_{A}, v_{A}\right\rangle(i=1,2, \ldots, n), \quad$ if $\omega=\left(\frac{1}{n}, \cdots \frac{1}{n}\right)$, it has $n \omega A_{i}=A$. By Eq. (8), taking note of $\sum_{i=1}^{n} w_{i}=1$, we obtain
Proof By Eq. (8) and the condition that $\left(A^{\prime}{ }_{1}, A^{\prime}{ }_{2}, \ldots A^{\prime}{ }_{n}\right)$ is any permutation of $\left(A_{1}, A_{2}, \ldots A_{n}\right)$, we can get the result directly.

\section{Selection of cold chain logistics enterprises under intuitionistic fuzzy environment}

Intuitionistic fuzzy multiple attribute decision making (IFMADM) problems are the process of choosing the best alternative from all of the possible alternatives which are evaluated by several attributes. A cold chain is a temperature-controlled supply chain, which is used to help

$$
\begin{aligned}
& \operatorname{GIFIHIA}_{\lambda}\left(A_{1}, A_{2}, \ldots A_{n}\right)=\left\langle\left(1-\prod_{i=1}^{n}\left(1-\left(1-v_{A}\right)^{\lambda}+\left(1-\left(u_{A}+v_{A}\right)\right)^{\lambda}\right)^{w_{i}}+\prod_{i=1}^{n}\left(1-\left(u_{A}+v_{A}\right)\right)^{\lambda \cdot w_{i}}\right)^{1 / \lambda}-\prod_{i=1}^{n}\left(1-\left(u_{A}+v_{A}\right)\right)^{w_{i}}\right. \\
&\left.1-\left(1-\prod_{i=1}^{n}\left(1-\left(1-v_{A}\right)^{\lambda}+\left(1-\left(u_{A}+v_{A}\right)\right)^{\lambda}\right)^{w_{i}}+\prod_{i=1}^{n}\left(1-\left(u_{A}+v_{A}\right)\right)^{\lambda w_{i}}\right)^{1 / \lambda}\right\rangle \\
&=\left\langle\left(1-\left(1-\left(1-v_{A}\right)^{\lambda}+\left(1-\left(u_{A}+v_{A}\right)\right)^{\lambda}\right)^{\sum_{i=1}^{n} w_{i}}+\left(1-\left(u_{A}+v_{A}\right)\right)^{\sum_{i=1}^{n} \lambda \cdot w_{i}}\right)^{1 / \lambda}\right. \\
&\left.-\left(1-\left(u_{A}+v_{A}\right)\right)^{\sum_{i=1}^{n} w_{i}} 1-\left(1-\left(1-\left(1-v_{A}\right)^{\lambda}+\left(1-\left(u_{A}+v_{A}\right)\right)^{\lambda}\right)^{\sum_{i=1}^{n} w_{i}}+\left(1-\left(u_{A}+v_{A}\right)\right)^{\sum_{i=1}^{n} \lambda w_{i}}\right)^{1 / \lambda}\right\rangle \\
&=\left\langle\left(1-\left(1-\left(1-v_{A}\right)^{\lambda}+\left(1-\left(u_{A}+v_{A}\right)\right)^{\lambda}\right)+\left(1-\left(u_{A}+v_{A}\right)\right)^{\lambda}\right)^{1 / \lambda}\right\rangle \\
&\left.-\left(1-\left(u_{A}+v_{A}\right)\right), 1-\left(1-\left(1-\left(1-v_{A}\right)^{\lambda}+\left(1-\left(u_{A}+v_{A}\right)\right)^{\lambda}\right)+\left(1-\left(u_{A}+v_{A}\right)\right)^{\lambda}\right)^{1 / \lambda}\right\rangle \\
&=\left\langle\left(\left(1-v_{A}\right)^{\lambda}\right)^{1 / \lambda}-\left(1-\left(u_{A}+v_{A}\right)\right), 1-\left(\left(1-v_{A}\right)^{\lambda}\right)^{1 / \lambda}\right\rangle=\left\langle u_{A}, v_{A}\right\rangle .
\end{aligned}
$$


extend and ensure the shelf life of products. Suppose that $X=\left\{x_{1}, x_{2}, \ldots, x_{n}\right\}$ is a set of cold chain logistics enterprises, $G=\left\{G_{1}, G_{2}, \ldots, G_{m}\right\}$ is a set of attributes with the associated weighting vector $w=\left(w_{1}, w_{2}, \ldots, w_{m}\right)^{T}$, satisfying $w_{i} \in\left[0,1\right.$ and $\sum_{i=1}^{m} w_{i}=1$.

Suppose that the evaluated values of the cold chain logistics enterprises $x_{i}(i=1,2, \ldots, n)$ under the attribute $G_{j}$ are represented by IFNs $A_{i j}=\left\langle u_{A_{i j}}, v_{A_{i j}}\right\rangle(i=1,2, \ldots$, $n j=1,2, \ldots, m)$, where $u_{A_{i j}}$ reflects the degree that the alternative $x_{i}(i=1,2, \ldots, n)$ satisfies the attribute $G=\left\{G_{1}, G_{2}, \ldots, G_{m}\right\} v_{A_{i j}}$ indicates the opposite meaning.

The construction of IFNs can refer to Pedrycz and Chen (2015), Lorkowski and Kreinovich (2015) and Naim and Hagras (2015).

Then the selection of cold chain logistics enterprises under intuitionistic fuzzy environment based on the GIFIHIA operator can be listed as follows.

Step 1 Determine the weights $w=\left(w_{1}, w_{2}, \ldots, w_{n}\right)^{T}$ and $\omega=\left(\omega_{1}, \ldots \omega_{n}\right)^{T}$, the meaning of $w$ and $\omega$ please refer to Definitions 4 and 5.

Step 2 Based on the GIFIHIA operator in Deinition 5, we aggregate the decision information by experts into collective ones, and the GIFIHIA operator is listed as Eq. (22). considered. For the sake of choosing the best cold chain logistics enterprise to cooperate, the foodstuff general corporation has brought a panel. After careful thinking of the alternatives and decision environment, five attributes $G=\left\{G_{1}, G_{2}, G_{3}, G_{4}, G_{5}\right\}$ are concluded to evaluate the ability of the candidate cold chain logistics enterprises.

- $G_{1}$ : Storage ability.

- $G_{2}$ : The levels of processing.

- $G_{3}$ : Transportation capability.

- $G_{4}$ : Logistics support capability

- $G_{5}$ : Coordinating optimization ability of business operations.

The evaluated decision information of the three possible alternatives under the above five attributes are presented by IFNs (Table 1).

Preliminary According to the method of constructing IFNs with granularity in Sect. 5, for example, experts mark her confidence by left membership value 2 and right membership value 5 on a scale from 1 to 10 to indicate the degrees $x_{1}$ satisfy the property $G_{1}$, thus $A_{11}$ in the numerical example is $\left\langle\underline{u_{A_{11}}}, 1-\overline{u_{A_{11}}}\right\rangle=\langle 2 / 10,1-5 / 10\rangle=$

$$
\begin{aligned}
& A_{i}=\operatorname{GIFIHIA}_{\lambda}\left(A_{i 1}, A_{i 2}, \ldots A_{i n}\right)=\left\langle\left( 1-\prod_{j=1}^{n}\left(1-\left(1-v_{\tilde{A}_{\text {index }(i)}}\right)^{\lambda}+\left(1-\left(u_{\tilde{A}_{\text {index }(i)}}+v_{\tilde{A}_{\text {index }(j)}}\right)\right)^{\lambda}\right)^{w_{j}}\right.\right. \\
& \left.+\prod_{j=1}^{n}\left(1-\left(u_{\tilde{A}_{\text {index }(j)}}+v_{\tilde{A}_{\text {index }(j)}}\right)\right)^{\lambda \cdot w_{j}}\right)^{1 / \lambda}-\prod_{j=1}^{n}\left(1-\left(u_{\tilde{A}_{\text {index }(i)}}+v_{\tilde{A}_{\text {index }(j)}}\right)\right)^{w_{j}}, \\
& \left.1-\left(1-\prod_{j=1}^{n}\left(1-\left(1-v_{\tilde{A}_{\text {index }(i j)}}\right)^{\lambda}+\left(1-\left(u_{\tilde{A}_{\text {index }(i)}}+v_{\tilde{A}_{\text {index }(i j)}}\right)\right)^{\lambda}\right)^{w_{j}}+\prod_{j=1}^{n}\left(1-\left(u_{\tilde{A}_{\text {index }(j)}}+v_{\tilde{A}_{\text {index }(j)}}\right)\right)^{\lambda w_{j}}\right)^{1 / \lambda}\right\rangle .
\end{aligned}
$$

Step 3 Rank the final IFNs $A_{i}(i=1,2, \ldots, n)$ by the score the accuracy functions in Definition 2.

Step 4 Rank the possible cold chain logistics enterprises $x_{i}(i=1,2, \ldots, n)$ and choose the best ones.

Step 5 Adjust the values of the parameter $\lambda$ according to decision makers' preference, and analyze the rakings of the cold chain logistics enterprises with different values of the parameter $\lambda$.

Step 6 Draw the figure of Step 5 to illustrate the selections of the cold chain logistics enterprises further.

\section{Numerical example}

Suppose that a foodstuff general corporation plan to choose a cold chain logistics enterprise to store and transport its goods. After thinking the market environment, three possible cold chain logistics enterprises $\left(\mathrm{x}_{1}, \mathrm{x}_{2}, \mathrm{x}_{3}\right)$ are to be
$<0.2,0.5>$. Similarly, we have $A_{i j}(i=1,2,3 ; j=1, \ldots, 5)$, and all evaluated IFNs are listed in Table 1.

Step 1 The weights $w$ and $\omega$ are given by the experts as $w$ $=(0.112,0.236,0.304,0.236,0.112)$ and $\omega=(0.2, \ldots 0.2)$.

Step 2 Suppose the induced values of $\tilde{A}_{i j}(i=1,2,3 ; j=1, \ldots, 5)$ given by the exsperts are $p_{i 1}=0.9$, $p_{i 2}=0.8, p_{i 3}=0.6, p_{i 4}=0.5, p_{i 5}=0.2 \quad(i=1,2,3)$, then by Eq. (22), and taking $\lambda=0.5$, we obtain

$A_{1}=$ GIFIHIA $_{0.5}\left(A_{11}, \ldots A_{15}\right)=\langle 0.4387,0.3262\rangle$.

Similarly, $A_{2}=\langle 0.4515,0.3625\rangle, A_{3}=\langle 0.3772,0.4413\rangle$.

Step 3 By the score function proposed by Chen and Tan (1994), we have

$S\left(A_{1}\right)=0.1125, S\left(A_{2}\right)=0.0891, S\left(A_{3}\right)=-0.0640$.

Step 4 By Definition 2, we have $S\left(A_{1}\right)>S\left(A_{2}\right)>S\left(A_{3}\right)$ and $x_{1}>x_{2}>x_{3}$. 
Table 1 Intuitionistic fuzzy matrix $\left(A_{i j}\right)_{3 \times 5}$

\begin{tabular}{llllll}
\hline & $G_{1}$ & $G_{2}$ & $G_{3}$ & $G_{4}$ & $G_{5}$ \\
\hline$x_{1}$ & $\langle 0.2,0.5\rangle$ & $\langle 0.4,0.2\rangle$ & $\langle 0.5,0.4\rangle$ & $\langle 0.3,0.3\rangle$ & $\langle 0.7,0.1\rangle$ \\
$x_{2}$ & $\langle 0.2,0.7\rangle$ & $\langle 0.6,0.3\rangle$ & $\langle 0.4,0.3\rangle$ & $\langle 0.4,0.4\rangle$ & $\langle 0.6,0.1\rangle$ \\
$x_{3}$ & $\langle 0.2,0.7\rangle$ & $\langle 0.4,0.3\rangle$ & $\langle 0.4,0.5\rangle$ & $\langle 0.3,0.4\rangle$ & $\langle 0.6,0.2\rangle$ \\
\hline
\end{tabular}

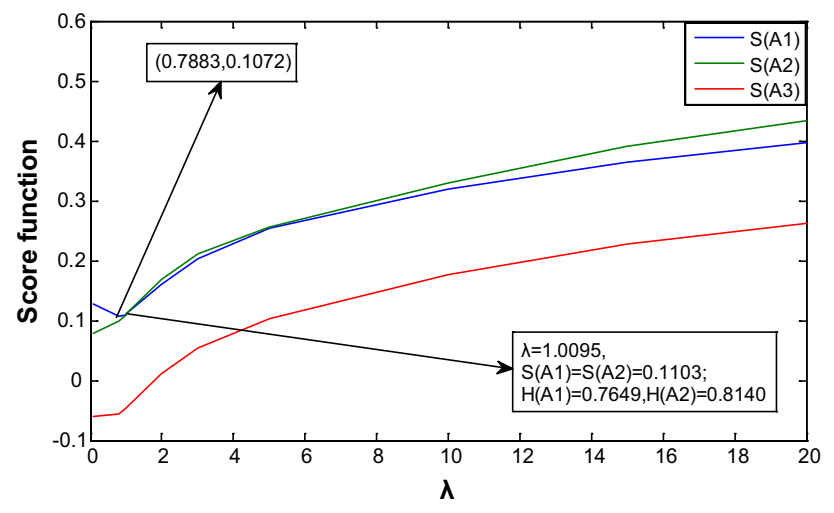

Fig. 1 Scores of the alternatives with different values of parameter $\lambda$

Step 5 Taking different values of the parameter $\lambda$ and $\lambda=0.001,0.002,0.003 \ldots, 20$, here we just list the scores and the rankings of involved alternatives with $\lambda=0.1,1,2,3,5,10,15,20$ as in Table 2 .

Step 6. The scores of invovled alternatives with different values of parameter $\lambda$ in Step 5 are shown in Fig. 1.

From Fig. 1, we find that $S\left(A_{1}\right)$ increases as $\lambda$ increases on $(0,0.7883]$, and decreases as $\lambda$ increases on $(0.7883,20]$. $S\left(A_{2}\right)$ and $S\left(A_{3}\right)$ increase as $\lambda$ increases on $(0,20]$. Moreover, the ranking of the alternatives with different values of the parameter $\lambda$ are concluded as follows.

1. When $\lambda \in(0,1.0095)$, the ranking of the three possible cold chain logistics enterprises is $x_{1}>x_{2}>x_{3}$. Therefore, $x_{1}$ is the best alternative.

2. When $\lambda \in(1.0095,20)$, the ranking of the three possible cold chain logistics enterprises is $x_{2}>x_{1}>x_{3}$. Thus, $x_{2}$ is the best alternative.

If we use the aggregation operators in Zhao et al. (2010) to aggregate a set of IFNs, when there exists only one non-membership degree of IFN equals to zero, the non-membership degree of aggregation result of $n$ IFNs is zero even if the non-membership degrees of $n-1$ IFNs are not zero, which is the weakness of the aggregation operators in Zhao et al. (2010). However, if we use the operators developed in this paper, the aggregation result can be explained reasonably. For example, Let $A_{1}=\langle 0.3,0.5\rangle, A_{2}=\langle 0.4,0.4\rangle$, $A_{3}=\langle 0.3,0.6\rangle, A_{4}=\langle 0.4,0.5\rangle, A_{5}=\langle 0.5,0\rangle$ be five IFNs, $w=\{0.25,0.20,0.15,0.18,0.22\}^{T}$ be the corresponding weight vector. By the generalized intuitionistic fuzzy weighted averaging (GIFWA) operator in Zhao et al. (2010), i.e.,

$$
\begin{gathered}
G I F W A_{\lambda}\left(A_{1}, A_{2}, \ldots A_{n}\right)=\left\langle\left(1-\prod_{i=1}^{n}\left(1-u_{A_{i}}{ }^{\lambda}\right)^{w_{i}}\right)^{1 / \lambda},\right. \\
\left.1-\left(1-\prod_{i=1}^{n}\left(1-\left(1-v_{A_{i}}{ }^{\lambda}\right)\right)^{w_{i}}\right)^{1 / \lambda}\right\rangle
\end{gathered}
$$

Taking $\lambda=0.5$, we get $G I F W A_{0.5}\left(A_{1}, A_{2}, \ldots A_{5}\right)=$ $\langle 0.3845,0\rangle$.

If we use the generalized intuitionistic fuzzy induced hybrid interaction averaging (GIFIHIA) operator in this paper, taking $\lambda=0.5$ and $\omega=(0.2, \ldots 0.2)^{T}$ for the convenience of comparison, we obtain

$\operatorname{GIFIHI}_{0.5}\left(A_{1}, A_{2}, \ldots A_{5}\right)=\langle 0.3758,0.4295\rangle$.

Obviously $\quad v_{\mathrm{GIFWA}_{0.5}\left(A_{1}, A_{2}, \ldots A_{5}\right)}=0, \quad$ while $v_{\text {GIFIHIA }_{0.5}\left(A_{1}, A_{2}, \ldots A_{5}\right)}=0.4295 \neq 0$, which shows that $v_{A_{5}}=0$ plays a decisive role by Eq. (23), While doesn't play a decisive role by Eq. (22). Therefore, the new generalized weighted operator developed by this paper is more practical from the averaging point of view.

The characteristics of GIFIHIA operator can be interpreted in the following five aspects.

1. It considers three relations of different IFNs: the interactions of membership function of different IFNs, the interactions of non-membership function of different IFN $s$ and the interactions between membership function and non-membership function of different IFNs.

2. It weights the IFNs $A_{i}=\left\langle u_{A_{i}}, v_{A_{i}}\right\rangle(i=1,2, \ldots, n)$ by the associated weights $\omega=\left(\omega_{1}, \ldots \omega_{n}\right)^{T}$ and multiplies

Table 2 Score function obtained by the GIFIHIA operator and the rank of the alternatives

\begin{tabular}{lllllllll}
\hline & GIFIHIA $_{0.1}$ & GIFIHIA $_{1}$ & GIFIHIA $_{2}$ & GIFIHIA $_{3}$ & GIFIHIA $_{5}$ & GIFIHIA $_{10}$ & GIFIHIA $_{15}$ & GIFIHIA $_{20}$ \\
\hline$x_{1}$ & 0.1281 & 0.1100 & 0.1596 & 0.2036 & 0.2535 & 0.3187 & 0.3640 & 0.3977 \\
$x_{2}$ & 0.0786 & 0.1098 & 0.1692 & 0.2117 & 0.2568 & 0.3306 & 0.3902 & 0.4344 \\
$x_{3}$ & -0.0593 & -0.0466 & 0.0119 & 0.0536 & 0.1031 & 0.1775 & 0.2277 \\
Ranking & $x_{1}>x_{2}>x_{3}$ & $x_{1}>x_{2}>x_{3}$ & $x_{2}>x_{1}>x_{3}$ & $x_{1}>x_{2}>x_{3}$ & $x_{2}>x_{1}>x_{3}$ & $x_{2}>x_{1}>x_{3}$ & $x_{2}>x_{1}>x_{3}$ & 0.2631 \\
\hline
\end{tabular}


these numbers by a balancing coefficient $n$, and then gets the weighted IFNs $n \omega_{i} A_{i}(i=1,2, \ldots, n)$.

3. It reorders the weighted IFNs $n \omega_{i} A_{i}(i=1,2, \ldots, n)$ according to the induced value $p_{i}(i=1,2, \cdots, n)$ as $\left(\tilde{A}_{\text {index }(1)}, \tilde{A}_{\text {index }(2)}, \ldots, \tilde{A}_{\text {index }(n)}\right)$.

4. Both the weighted IFNs $w_{i} \tilde{A}_{\text {index }(i)}(i=1,2, \ldots, n)$ and their induced value $p_{i}(i=1,2, \ldots, n)$ are considered, and all the IFNs $A_{i}(i=1,2, \ldots, n)$ are aggregated into a collective one.

5. The attitude of decision makers are considered by taking different values of $\lambda$ according to decision makers' preferences.

\section{Conclusions}

In this paper, we present the IFIHIA operator and the GIFIHIA operator, taking the interactions of different IFNs into consideration, reordering the involved IFNs according to the induced values and then aggregate them into a collective one, considering the attitudes of decision makers by taking different values of parameter according to decision makers' preferences. We investigate the properties of these operators and apply them to the selection of cold chain logistics enterprises under intuitionistic fuzzy environment. Examples are illustrated to show the validity and feasibility of the new approach. We also give some comparisons between this paper and other papers.

In the succeeding work, we will develop a class of fuzzy numbers intuitionistic fuzzy hybrid interaction averaging operators based on the existing works and apply them to the selection of cold chain logistics enterprises, decision support, recommender systems and multiple attribute group decision making.

\section{References}

Antonelli M, Ducange P, Lazzerini B, Marcelloni F (2016) Multiobjective evolutionary design of granular rule-based classifiers. Granul Comput 1(1):37-58

Apolloni B, Bassis S, Rota J, Galliani GL, Gioia M, Ferrari L (2016) A neuro fuzzy algorithm for learning from complex granules. Granul Comput 1(4):225-246

Atanassov KT (1986) Intuitionistic fuzzy sets. Fuzzy Sets Syst 20(1):87-96

Atanassov KT (1994) New operations defined over the intuitionistic fuzzy sets. Fuzzy Sets Syst 61(2):137-142

Beliakov G, James S, Mordelová J, Rückschlossová T, Yager RR (2010) Generalized Bonferroni mean operators in multi-criteria aggregation. Fuzzy Sets Syst 161(17):2227-2242

Chen TY (2014) A prioritized aggregation operator-based approach to multiple criteria decision making using interval-valued intuitionistic fuzzy sets: a comparative perspective. Inform Sci 281:97-112
Chen SM, Chang CH (2015) A novel similarity measure between Atanassov's intuitionistic fuzzy sets based on transformation techniques with applications to pattern recognition. Inform Sci 291:96-114

Chen SM, Tan JM (1994) Handling multicriteria fuzzy decisionmaking problems based on vague set theory. Fuzzy Sets Syst 67(2):163-172

Chen SM, Tsai WH (2016) Multiple attribute decision making based on novel interval-valued intuitionistic fuzzy geometric averaging operators. Inform Sci 367:1045-1065

Chen SM, Cheng SH, Lan TC (2016a) A novel similarity measure between intuitionistic fuzzy sets based on the centroid points of transformed fuzzy numbers with applications to pattern recognition. Inform Sci 343:15-40

Chen SM, Cheng SH, Lan TC (2016b) Multicriteria decision making based on the TOPSIS method and similarity measures between intuitionistic fuzzy values. Inform Sci 367-368:279-295

Ciucci D (2016) Orthopairs and granular computing. Granul Comput 1(3):159-170

Das S, Kar S, Pal T (2017) Robust decision making using intuitionistic fuzzy numbers. Granul Comput. doi:10.1007/ s41066-016-0024-3

De SK, Biswas R, Roy AR (2000) Some operations on intuitionistic fuzzy sets. Fuzzy Sets Syst 114(3):477-484

Dubois D, Prade H (2016) Bridging gaps between several forms of granular computing. Granul Comput 1(2):115-126

He Y, Chen H, Zhou L, Liu J, Tao Z (2013) Generalized intervalvalued Atanassov's Intuitionistic fuzzy power operators and their application to multiple attribute group decision making. Int J Fuzzy Syst 15:401-441

He Y, Chen H, Zhou L, Liu J, Tao Z (2014a) Intuitionistic fuzzy geometric interaction averaging operators and their application to multi-criteria decision making. Inform Sci 259:142-159

He Y, Chen H, Zhou L, Han B, Zhao Q, Liu J (2014b) Generalized intuitionistic fuzzy geometric interaction operators and their application to decision making. Expert Syst Appl 41:2484-2495

He Y, He Z, Chen H (2015) Intuitionistic fuzzy interaction Bonferroni means and its application to multiple attribute decision making. IEEE Trans Cybern 45(1):116-128

He Y, He Z, Deng Y, Zhou P (2016) IFPBMs and their application to multiple attribute group decision making. J Oper Res Soc 67(1):127-147

Hong DH, Choi CH (2000) Multicriteria fuzzy decision-making problems based on vague set theory. Fuzzy Sets Syst 114(1):103-113

Kovalerchuk B, Kreinovich V (2017) Concepts of solutions of uncertain equations with intervals, probabilities and fuzzy sets for applied tasks. Granul Comput. doi:10.1007/s41066-016-0031-4

Kreinovich V (2016) Solving equations (and systems of equations) under uncertainty: how different practical problems lead to different mathematical and computational formulations. Granul Comput 1(3):171-179

Lingras P, Haider F, Triff M (2016) Granular meta-clustering based on hierarchical, network, and temporal connections. Granul Comput 1(1):71-92

Liu H, Cocea M (2017) Granular computing based approach for classification towards reduction of bias in ensemble learning. Granul Comput. doi:10.1007/s41066-016-0034-1

Liu H, Gegov A, Cocea M (2016) Rule-based systems: a granular computing perspective. Granul Comput 1(4):259-274

Livi L, Sadeghian A (2016) Granular computing, computational intelligence, and the analysis of non-geometric input spaces. Granul Comput 1(1):13-20 
Loia V, D'Aniello, G, Gaeta A, Orciuoli F (2016) Enforcing situation awareness with granular computing: a systematic overview and new perspectives. Granul Comput 1(2):127-143

Lorkowski J, Kreinovich V (2015) Granularity helps explain seemingly irrational features of human decision making[M] granular computing and decision-making, vol 1. Springer International Publishing, New York, pp 1-31

Maciel L, Ballini R, Gomide F (2016) Evolving granular analytics for interval time series forecasting. Granul Comput 1(4):213-224

Mendel JM (2016) A comparison of three approaches for estimating (synthesizing) an interval type-2 fuzzy set model of a linguistic term for computing with words. Granul Comput 1(1):59-69

Merigó J, Gil-Lafuente A, Zhou L, Chen H (2011) Generalization of the linguistic aggregation operator and its application in decision making. J Syst Eng Electron 22:593-603

Min F, Xu J (2016) Semi-greedy heuristics for feature selection with test cost constraints. Granul Comput 1(3):199-211

Naim S, Hagras H (2015) A Type-2 fuzzy logic approach for multicriteria group decision making, vol 1. Springer International Publishing, New York, pp 123-164

Pedrycz W, Chen SM (2015) Granular computing and decision-making: interactive and iterative approaches. Springer, Heidelberg

Peters G, Weber R (2016) DCC: a framework for dynamic granular clustering. Granul Comput 1(1):1-11

Rodriguez RM, Martinez L, Herrera F (2012) Hesitant fuzzy linguistic term sets for decision making. IEEE Trans Fuzzy Syst 20(1):109-119

Rodríguez RM, MartıNez L, Herrera F (2013) A group decision making model dealing with comparative linguistic expressions based on hesitant fuzzy linguistic term sets. Inform Sci 241:28-42

Rodríguez RM, Martínez L, Torra V, Xu ZS, Herrera F (2014) Hesitant fuzzy sets: state of the art and future directions. Int J Intell Syst 29(6):495-524

Sanchez MA, Castro JR, Castillo O, Mendoza O, Rodriguez-Diaz A, Melin P (2017) Fuzzy higher type information granules from an uncertainty measurement. Granular. Computing. doi:10.1007/ s41066-016-0030-5

Skowron A, Jankowski A, Dutta S (2016) Interactive granular computing. Granul Comput 1:95-113

Song M, Wang Y (2016) A study of granular computing in the agenda of growth of artificial neural networks. Granular. Computing 1(4):247-257
Wang G, Yang J, Xu J (2017) Granular computing: from granularity optimization to multi-granularity joint problem solving. Granul Comput. doi:10.1007/s41066-016-0032-3

Wei G (2012) Hesitant fuzzy prioritized operators and their application to multiple attribute decision making. Knowl Based Syst 31: $176-182$

Wei G, Zhao X (2012) Some induced correlated aggregating operators with intuitionistic fuzzy information and their application to multiple attribute group decision making. Expert Syst Appl 39(2):2026-2034

Wilke G, Portmann E (2016) Granular computing as a basis of human-data interaction: A cognitive cities use case. Granul Comput 1(3):181-197

$\mathrm{Xu} \mathrm{Z}$ (2007) Intuitionistic fuzzy aggregation operations. IEEE Trans Fuzzy Syst 15:1179-1187

Xu Z, Gou X (2017) An overview of interval-valued intuitionistic fuzzy information aggregations and applications. Granul Comput. doi:10.1007/s41066-016-0023-4

$\mathrm{Xu}$ Z, Wang H (2016) Managing multi-granularity linguistic information in qualitative group decision making: an overview. Granul Comput 1(1):21-35

Xu Z, Xia M (2011) Induced generalized intuitionistic fuzzy operators. Knowl Based Syst 24(2):197-209

$\mathrm{Xu} \mathrm{Z}$, Yager R (2006) Some geometric aggregation operators based on intuitionistic fuzzy sets. Int J Gen Syst 35(4):417-433

Yager R (2008) Prioritized aggregation operators. Int J Approx Reason 48(1):263-274

Yager R, Filev D (1999) Induced ordered weighted averaging operators. IEEE Trans Syst Man Cybern Part B Cybern 29(2):141-150

Yao Y (2016) A triarchic theory of granular computing. Granul Comput $1: 145-157$

Ye J (2010) Fuzzy decision-making method based on the weighted correlation coefficient under intuitionistic fuzzy environment. Eur J Oper Res 205:202-204

Zadeh LA (1965) Fuzzy sets. Inf Control 8(3):338-353

Zhao H, Xu Z, Ni M, Liu S (2010) Generalized aggregation operators for intuitionistic fuzzy sets. Int J Intell Syst 25(1):1-30

Zhou X (2017) Membership grade mining of mutually inverse fuzzy implication propositions. Granul Comput. doi:10.1007/ s41066-016-0033-2 